\title{
Computational Modeling Suggests That Response Properties Rather Than Spatial Position Determine Connectivity Between Olfactory Glomeruli
}

\author{
Christiane Linster, ${ }^{1}$ Silke Sachse, ${ }^{2}$ and C. Giovanni Galizia ${ }^{3}$ \\ ${ }^{1}$ Department of Neurobiology and Behavior, Cornell University, Ithaca, New York; ${ }^{2}$ The Rockefeller \\ University, New York, New York; and ${ }^{3}$ Department of Entomology, University of California, Riverside, California
}

Submitted 15 December 2004; accepted in final form 19 January 2005

\begin{abstract}
Linster, Christiane, Silke Sachse, and C. Giovanni Galizia. Computational modeling suggests that response properties rather than spatial position determine connectivity between olfactory glomeruli. $J$ Neurophysiol 93: 3410-3417, 2005. First published January 26, 2005; doi:10.1152/jn.01285.2004. Olfactory responses require the representation of high-dimensional olfactory stimuli within the constraints of two-dimensional neural networks. We used a computational model of the honeybee antennal lobe to test how inhibitory interactions in the antennal lobe should be organized to best reproduce the experimentally measured input-output function in this structure. Our simulations show that a functionally organized inhibitory network, as opposed to an anatomically or all-to-all organized inhibitory network, best reproduces the input-output function of the antennal lobe observed with calcium imaging. In this network, inhibition between each pair of glomeruli was proportional to the similarity of their odor-response profiles. We conclude that contrast enhancement between odorants in the honeybee antennal lobe is best achieved when interglomerular inhibition is organized based on glomerular odor response profiles rather than on anatomical neighborhood relations.
\end{abstract}

\section{IN T R O D U C T I O N}

Olfactory representations are intrinsically high dimensional yet must be represented within morphologically two-dimensional neural networks. A great deal of research in olfaction has focused on the representation of olfactory information within the first processing stage, the olfactory bulb (OB) or antennal lobe (AL) glomeruli, particularly with respect to how chemical stimuli are mapped onto olfactory glomeruli and how this mapping may relate to their perceptual qualities (Galizia and Menzel 2001; Linster et al. 2001; Xu et al. 2000). While a number of studies have illustrated the importance of inhibitory networks within the OB or AL for the shaping and processing of olfactory information (Stopfer et al. 1997; Urban 2002; Yokoi et al. 1995), it is not clear how exactly these inhibitory networks are organized to provide filtering and contrast enhancement capabilities. It is generally believed that odors are represented as combinatorial activity patterns across olfactory glomeruli; odor-specific spatiotemporal activity patterns in olfactory glomeruli have been shown in vertebrates (Meister and Bonhoeffer 2001; Rubin and Katz 1999; Wachowiak et al. 2000, 2002) and invertebrates (Christensen et al. 2000; Faber et al. 1999; Galizia and Menzel 2000; Hansson et al. 2003; Joerges et al. 1997; Sachse et al. 1999). It has been suggested that the broadly distributed activation patterns observed at the glomerular layer can be modulated by the OB/AL network in such a way as to ensure specific discrimination between chem-

Address for reprint requests and other correspondence: C. Linster, Cornell University, Mudd Hall, Ithaca, NY 14853 (E-mail: CL243@cornell.edu). ically similar odorants (Duchamp-Viret and Duchamp 1993; Linster and Gervais 1996; Linster and Hasselmo 1997; Meredith 1986; Sachse and Galizia 2002; Urban 2002). Specifically, using calcium imaging in the AL of honeybees, we have recently shown that odor representations at the output of the AL network are considerably more distinct than those at the input (Sachse and Galizia 2003; but see Ng et al. 2002; Wang et al. 2003; Wilson et al. 1994 for data suggesting either no change between input and output response profile or broadening in fruit flies). Because of the extensive inhibitory network within the $\mathrm{OB}$ and $\mathrm{AL}$, as well as the effects of inhibitory blockers on odor discrimination (Sachse and Galizia 2002; Stopfer et al. 1997), it is clear that this inhibitory network is at least partially responsible for the modulation of olfactory representations within the AL/OB. Our ability to directly compare the processed odor representations at the output of the AL network to the corresponding input information (mediated by olfactory sensory neurons to the AL glomeruli) presents us with the unique opportunity to ask how the inhibitory processes within this network should be organized to compute this specific input-output function (Sachse and Galizia 2003). Using a computational model (Cleland and Linster 2002; Linster and Smith 1997), we here show that in the honeybee AL, a functional rather than anatomical organization of the inhibitory network is necessary to produce the experimentally measured transformation between input and output activation, contrary to common assumptions. In contrast to previous computational models of the olfactory system, we here base our conclusions on experimentally measured odor responses.

\section{E THODS}

\section{Neurons and connections}

Similar to our previous models of the honeybee AL (Linster and Masson 1996; Linster and Smith 1997), the model incorporates olfactory sensory neurons (OSNs), AL interneurons (LNs), and AL projection neurons (PNs). The evolution of each neuron's membrane potential $v(t)$ around its resting potential is described by a first-order differential equation

$$
\tau \frac{\mathrm{d} v(\mathrm{t})}{\mathrm{d} t}+v(t)=\mathrm{I}_{\mathrm{ext}}(t)
$$

where $\tau$ is the charging time constant of the neuron and $I_{\text {ext }}(t)$ is the total input current at time $t$. For OSNs, the net total input is directly proportional to the olfactory stimulation that they receive. For LNs

\footnotetext{
The costs of publication of this article were defrayed in part by the payment of page charges. The article must therefore be hereby marked "advertisement" in accordance with 18 U.S.C. Section 1734 solely to indicate this fact.
} 
and PNs, the input from a particular synapse at time $t$ is computed as a function of the connection strength $w_{i j}$ between the presynaptic neurons $j$ and the postsynaptic neuron $i$, the conductance change $g(t)$ due to a presynaptic event $x_{j}\left(t_{0}\right)$ at time $t_{0}$, and the difference between the Nernst potential $E_{N, i j}$ of the associated channel type and the current membrane potential $v_{i}(t)$ of the postsynaptic neuron

$$
I_{\text {ext }, i j}(t)=W_{i j} g\left(X_{j}\left(t_{0}\right)\right)\left[E_{N, i j}-V_{\mathrm{i}}(t)\right]
$$

The time course of the conductance change $g(t)$ is described by a double-exponential function

$$
g(t)=X_{i}\left(t_{0}\right) g_{\max } \frac{\tau_{1} \tau_{2}}{\tau_{1}-\tau_{2}}\left(e^{-\left(t-t_{0}\right) / \tau_{1}}-e^{-\left(t-t_{0}\right) / \tau_{2}}\right)
$$

In the model, functionally similar populations of OSNs and LNs are represented by a single equation approximating their average firing frequencies. Their (analog) output activities are modeled by a linear threshold function with saturation, representing the average firing activity of a large number of neurons, as shown in Eq. 4. In contrast, the model PNs were individually simulated; consequently, they are generating action potentials. At each time step $t$ (sampling step $=1$ $\mathrm{ms})$, the probability $P$ for a spike to occur at a $\mathrm{PN}^{\prime}$ s output $x(t)$ was modeled as a linear threshold function with saturation of the membrane potential

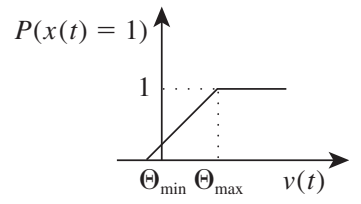

where $\theta_{\min }$ is the spiking threshold and $\theta_{\max }$ is the saturation threshold (at which the maximum spike rate occurs). After any occurrence of a spike, the membrane potential was reset to the resting potential. The differential equations were iterated using exponential Euler with a time step of $1 \mathrm{~ms}$.

\section{Model architecture}

The computational model of the honeybee AL presented here was designed to incorporate and further investigate results from calcium imaging obtained in the AL. The basic architecture of the model, designed to replicate some of the main known features of AL anatomy and physiology, has been adapted from our previous models (Cleland and Linster 2002; Linster et al. 1994; Linster and Cleland 2001; Linster and Masson 1996; Linster and Smith 1997). One of the major adjustments made in the present version of the model is the simulation of "real" input patterns for each odor stimulus as directly extracted from calcium-imaging data. Consequently, we could simulate real odorant response patterns instead of constructing fictional odorant representations in a hypothetical multi-dimensional space as is usually done. Existing data-derived features of the model, e.g., its fieldoscillatory properties and their dependence on inhibitory interactions, have been carefully preserved in this version (Cleland and Linster 2002; Stopfer et al. 1997). We here focus on the question of how inhibitory connections should be organized to best replicate the measured input-output function of the AL (Sachse and Galizia 2003). The model serves to test how the transformation from input (glomerular input pattern) to output (glomerular output pattern), as measured in calcium-imaging experiments, can best be achieved by the AL neural network.

In the honeybee AL, all synaptic interactions are located in areas of high synaptic density called glomeruli (Gascuel and Masson 1991). In the present model, the number of these glomeruli is reduced from 160 to 20 , corresponding to the number of glomeruli that could be visualized and for which calcium-imaging data had been obtained from both the input and output (PNs) in response to a common set of odorants (Sachse and Galizia 2002, 2003; Sachse et al. 1999) (see Fig. $1 A$ for detailed description of the model architecture). Each model glomerulus received input from one sensory neuron (OSN, representing the summed activity of many convergent olfactory sensory neurons) and contained one local interneuron which receives excitatory input in that glomerulus and inhibits all other glomeruli [called heteroLN by Fonta et al. (1993), Fig. 1B], also representing the summed activity of many functionally attuned neurons and one PN. A second class of local interneurons [called homoLN by Fonta et al. (1993)] is included in the model; these homoLNs receive input in all glomeruli, project heavily onto themselves, and inhibit all other LNs and all PNs in a homogeneous manner (not shown in Fig. 1A). The feedback connections of these homoLNs onto themselves are likely to provide the oscillatory dynamics that have been observed experimentally (Stopfer et al. 1997). When stimulated with simulated odorants, the AL network exhibited oscillatory dynamics similar to those described experimentally and PN spiking was phase-locked with the population oscillation. The oscillatory dynamics and phase-locking in the model are dependent on homoLN feedback inhibitory connections (Cleland and Linster 2002) and therefore disappear when these inhibitory interactions are blocked as was shown experimentally when the GABAergic antagonist picrotoxin was introduced into the AL (MacLeod and Laurent 1996; Stopfer et al. 1997).
A

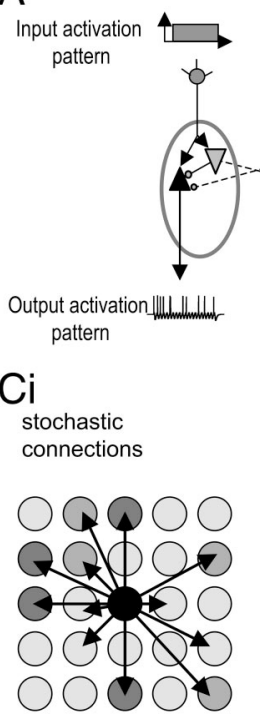

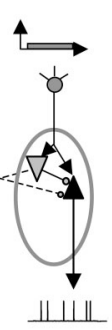

OSN

$\triangle \mathrm{PN}$

$\nabla$ heteroLN

$\nabla$ Excitatory synapse

- Inhibitory synapse

Cii morphological connections

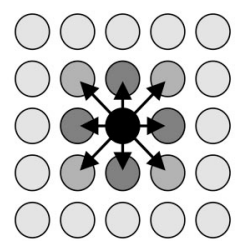

B

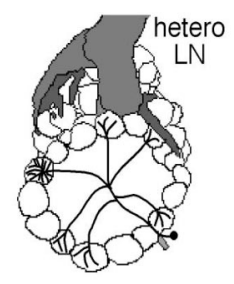

Ciii functional connections

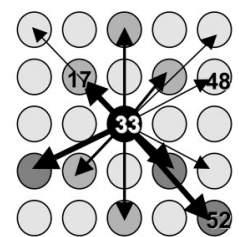

FIG. 1. Schematic depiction of antennal lobe model. $A$ : simulated olfactory sensory neurons (OSNs), which are differentially activated by odorants, project to 1 glomerulus each. Within this glomerulus, they make excitatory synapses onto local interneurons (heteroLN) and PN. Within each glomerulus, heteroLNs, each of which represents a population of similarly activated LNs, inhibit the local PN. These heteroLNs also project to other glomeruli in the network in which they connect to PNs (--). The pattern of heteroLN projections between glomeruli is the antennal lobe (AL) characteristic under investigation in this report; all other parameters were chosen in uniform distributions around the fixed mean values detailed in Table 1. A 2nd class of interneurons (not shown here), homoLNs, receives input from OSNs in all glomeruli, feeds back heavily onto itself and inhibits PNs and LNs uniformly in all glomeruli. $B$ : illustration of the heteroLN type of interneuron thought to provide interglomerular inhibition. HeteroLNs have dense arborizations in 1 glomerulus in addition to sparser glomerular arborizations throughout the antennal lobe. $C, i-i i i$ : schematic representation of the 3 types of interglomerular inhibitory networks modeled here. $\mathrm{Ci}$, stochastic connections: all glomeruli inhibit each other with the same, variable, probability. Cii, morphological connections: glomeruli inhibit their morphological neighbors. Ciii, functional connections: glomeruli inhibit each other proportionally to the similarity in their odor-response profiles. 
TABLE 1. Correlation values $c$ between glomerular odor responses as calculated from calcium imaging data of 60 odorants

\begin{tabular}{|c|c|c|c|c|c|c|c|c|c|c|c|c|c|c|c|c|c|c|c|c|}
\hline & 17 & 18 & 23 & 24 & 25 & 28 & 29 & 30 & 33 & 35 & 36 & 37 & 38 & 42 & 47 & 48 & 49 & 52 & 60 & 62 \\
\hline 17 & & & & & & & & & & & & & & & & & & & & \\
\hline 18 & 0.47 & & & & & & & & & & & & & & & & & & & \\
\hline 23 & 0.47 & 0.49 & & & & & & & & & & & & & & & & & & \\
\hline 24 & 0.66 & 0.39 & 0.70 & & & & & & & & & & & & & & & & & \\
\hline 25 & 0.22 & 0.25 & 0.86 & 0.53 & & & & & & & & & & & & & & & & \\
\hline 28 & 0.35 & 0.13 & 0.70 & 0.56 & 0.72 & & & & & & & & & & & & & & & \\
\hline 29 & 0.19 & 0.26 & 0.77 & 0.38 & 0.81 & 0.66 & & & & & & & & & & & & & & \\
\hline 30 & 0.26 & 0.42 & 0.49 & 0.52 & 0.48 & 0.47 & 0.46 & & & & & & & & & & & & & \\
\hline 33 & 0.69 & 0.50 & 0.66 & 0.76 & 0.44 & 0.50 & 0.52 & 0.55 & & & & & & & & & & & & \\
\hline 35 & 0.32 & 0.35 & 0.82 & 0.54 & 0.83 & 0.77 & 0.85 & 0.58 & 0.56 & & & & & & & & & & & \\
\hline 36 & 0.31 & 0.19 & 0.73 & 0.53 & 0.76 & 0.87 & 0.79 & 0.53 & 0.61 & 0.79 & & & & & & & & & & \\
\hline 37 & 0.21 & 0.35 & 0.81 & 0.58 & 0.77 & 0.58 & 0.74 & 0.47 & 0.53 & 0.72 & 0.66 & & & & & & & & & \\
\hline 38 & 0.20 & 0.09 & 0.68 & 0.45 & 0.80 & 0.77 & 0.80 & 0.54 & 0.42 & 0.84 & 0.76 & 0.65 & & & & & & & & \\
\hline 42 & 0.29 & 0.34 & 0.75 & 0.55 & 0.66 & 0.62 & 0.77 & 0.59 & 0.65 & 0.75 & 0.83 & 0.76 & 0.65 & & & & & & & \\
\hline 47 & 0.15 & 0.17 & 0.54 & 0.30 & 0.53 & 0.50 & 0.63 & 0.64 & 0.39 & 0.68 & 0.64 & 0.52 & 0.64 & 0.75 & & & & & & \\
\hline 48 & 0.00 & 0.04 & 0.38 & 0.08 & 0.48 & 0.42 & 0.57 & 0.47 & 0.22 & 0.57 & 0.48 & 0.46 & 0.65 & 0.55 & 0.78 & & & & & \\
\hline 49 & 0.01 & 0.28 & 0.63 & 0.32 & 0.73 & 0.46 & 0.75 & 0.51 & 0.39 & 0.71 & 0.60 & 0.75 & 0.72 & 0.68 & 0.65 & 0.74 & & & & \\
\hline 52 & 0.32 & 0.21 & 0.61 & 0.55 & 0.62 & 0.71 & 0.70 & 0.64 & 0.61 & 0.75 & 0.84 & 0.56 & 0.72 & 0.82 & 0.72 & 0.50 & 0.58 & & & \\
\hline 60 & 0.10 & 0.07 & 0.45 & 0.21 & 0.55 & 0.45 & 0.49 & 0.53 & 0.30 & 0.54 & 0.55 & 0.40 & 0.51 & 0.58 & 0.84 & 0.76 & 0.60 & 0.57 & & \\
\hline 62 & 0.11 & 0.18 & 0.45 & 0.33 & 0.51 & 0.45 & 0.52 & 0.51 & 0.46 & 0.58 & 0.58 & 0.46 & 0.57 & 0.66 & 0.79 & 0.72 & 0.69 & 0.70 & 0.85 & \\
\hline
\end{tabular}

In the simulations testing functional inhibition, these values were used to determine the inhibitory connection strength $c^{*} w$ between pairs of glomeruli. Data from Galizia et al. (1999) and Sachse et al. (1999).

The model heteroLNs, providing interglomerular inhibition, were based on morphological data (Fonta et al. 1993). Morphological studies in cockroach have suggested that heteroLNs have both input and output synapses within their principal glomerulus (Malun 1991a,b), whereas they may have primarily output synapses in the other glomeruli they invade. In the model, heteroLNs receive excitatory inputs from OSNs within their "principal" (densely invaded) glomerulus. In addition to these intraglomerular connections, heteroLNs have sparse arborizations in glomeruli all over the AL (Fonta et al. 2002) that are assumed to synapse on PNs in these secondary glomeruli. The effect of interglomerular inhibitory connections onto PNs by heteroLNs is the main focus of this report. We have investigated three different interglomerular projection patterns: stochastic distributions of inhibitory connections among glomeruli (Fig. 1Ci), morphological projections, in which inhibitory connections are made between anatomically neighboring glomeruli (Fig. 1Cii), and functional inhibitory projections between glomeruli that display similar responses to odorants (Fig. 1Ciii, Table 1). For each simulation, a new network was created; all parameter values (listed in Table 2) were chosen randomly in uniform distribution $( \pm 10 \%)$ around the indicated mean values, thus ensuring that our results were not due to a specific choice of parameters.

\section{R E S U L T S}

In the model, glomerular sensitivities to odorants were directly derived from published glomerular calcium-imaging data (bath applied) (Sachse et al. 1999). In those experiments, the brain was stained by superfusing it with the calciumsensitive dye calcium green AM. Changes in intracellular calcium concentration within glomeruli were measured as responses to odor stimulation. Because the dye was bathapplied, the precise cellular origin of these signals is unknown. However, the response magnitude is a likely estimate of sensory neuron response magnitude, as discussed by Sachse and Galizia (2003). These activities are referred to as glomerular input pattern in this paper. Activation values have been normalized for each odorant to enable comparison between animals; consequently, each odorant is represented by its average relative glomerular activation pattern (varying between 0 and 1; see Fig. 2, glomerular input pattern). In the model, these activation patterns form the input and are conveyed to the neurons in each glomerulus via the OSNs. A

TABLE 2. Simulation parameters

\begin{tabular}{|c|c|c|c|c|}
\hline Neurons & \multicolumn{2}{|c|}{ Membrane Time Constants } & Theta min & Theta max \\
\hline $\begin{array}{l}\text { Olfactory sensory neurons (OSN) } \\
\text { Local interneurons (LN) } \\
\text { Projection neurons (PN) }\end{array}$ & $\begin{array}{l}\tau=5.0 \mathrm{~ms} \\
\tau=15.0 \mathrm{~ms} \\
\tau=4.0 \mathrm{~ms}\end{array}$ & $\begin{array}{l}\theta_{\min }=0.0 \\
\theta_{\min }=0.2 \\
\theta_{\min }=-0.1\end{array}$ & $\begin{array}{l}\theta_{\max }=1.0 \\
\theta_{\max }=2.0 \\
\theta_{\max }=10.0\end{array}$ & \\
\hline Synapses & Synaptic Weights & Reversal Potential & Rise Time & Fall Time \\
\hline $\begin{array}{l}\text { Afferent, OSN to PN } \\
\text { Afferent, OSN to heteroLN } \\
\text { Afferent, OSN to homoLN } \\
\text { Local inhibitory, heteroLN to PN } \\
\text { Local inhibitory, homoLN to PN } \\
\text { Local inhibitory, homoLN to heteroLN } \\
\text { homoLN inhibitory feedback } \\
\text { Interglomerular, heteroLN to PN }\end{array}$ & $\begin{array}{l}\mathrm{W}_{\text {OSN-PN }}=0.02 \\
\mathrm{~W}_{\text {OSN-LN }}=0.01 \\
\mathrm{~W}_{\text {OSN-hLN }}=0.02 \\
\mathrm{~W}_{\text {LN-PN }}=0.005 \\
\mathrm{~W}_{\mathrm{hLN-PN}}=0.02 \\
\mathrm{~W}_{\mathrm{hLN-LN}}=0.02 \\
\mathrm{~W}_{\mathrm{hLN-hLN}}=1.0 \\
\mathrm{~W}_{\mathrm{LN-PN}}=w / 10\end{array}$ & $\begin{array}{l}\mathrm{E}_{\mathrm{N}, \mathrm{OSN}-\mathrm{PN}}=70 \\
\mathrm{E}_{\mathrm{N}, \mathrm{OSN}-\mathrm{LN}}=-70 \\
\mathrm{E}_{\mathrm{N}, \mathrm{OSN}-\mathrm{hLN}}=-70 \\
\mathrm{E}_{\mathrm{N}, \mathrm{LN}-\mathrm{PN}}=-10 \\
\mathrm{E}_{\mathrm{N}, \mathrm{hLN}-\mathrm{PN}}=-10 \\
\mathrm{E}_{\mathrm{N}, \mathrm{hLN}-\mathrm{LN}}=-10 \\
\mathrm{E}_{\mathrm{N}, \mathrm{hLN}-\mathrm{hLN}}=-10 \\
\mathrm{E}_{\mathrm{N}, \mathrm{LN}-\mathrm{PN}}=-10\end{array}$ & $\begin{array}{l}\tau_{1}=1.0 \\
\tau_{1}=1.0 \\
\tau_{1}=1.0 \\
\tau_{1}=4.0 \\
\tau_{1}=4.0 \\
\tau_{1}=4.0 \\
\tau_{1}=4.0 \\
\tau_{1}=4.0\end{array}$ & $\begin{array}{l}\tau_{2}=2.0 \\
\tau_{2}=1.0 \\
\tau_{2}=1.0 \\
\tau_{2}=8.0 \\
\tau_{2}=8.0 \\
\tau_{2}=8.0 \\
\tau_{2}=8.0 \\
\tau_{2}=8.0\end{array}$ \\
\hline
\end{tabular}

Note that for each simulation, a new network was created; all parameter values were chosen randomly in uniform distribution ( $\pm 10 \%)$ around the indicated mean values, thus ensuring that our results were not due to a specific choice of parameters. See Eqs. 2 and 3 for stimulation parameters. 


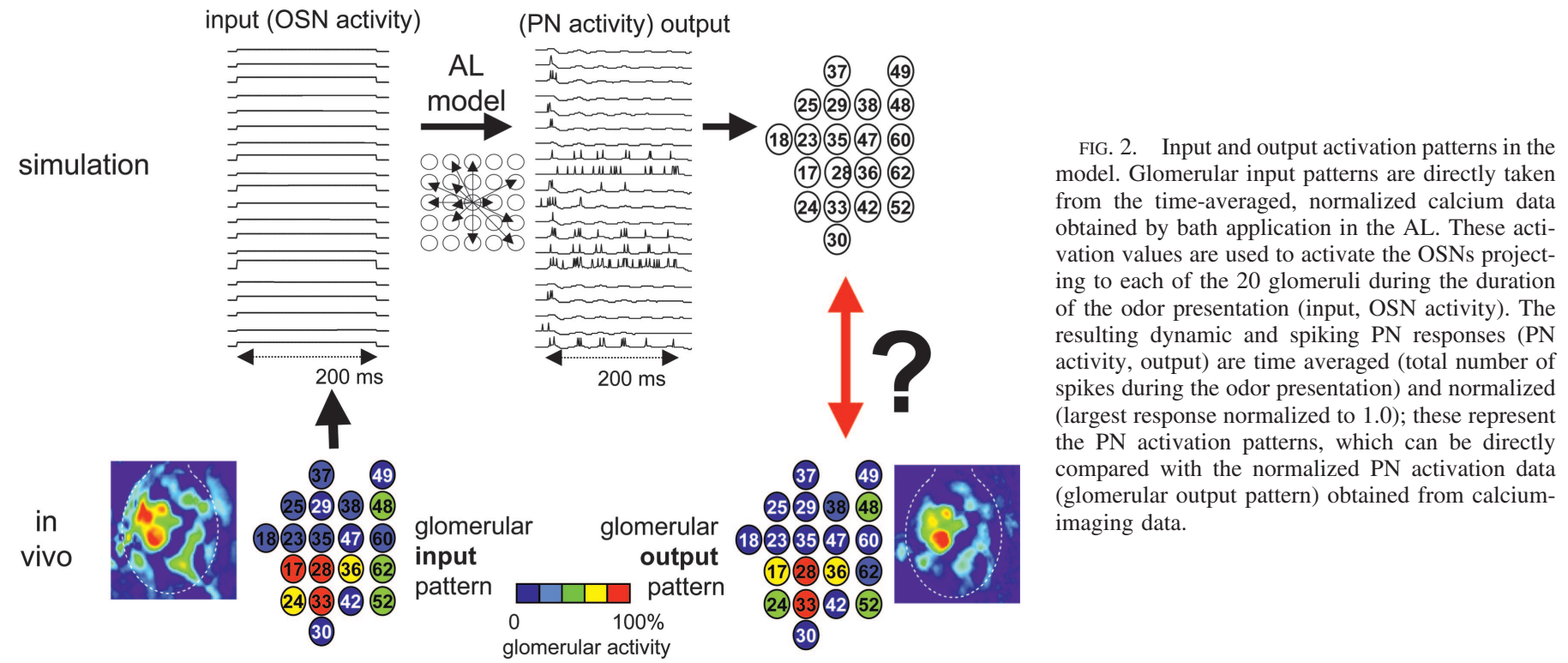

constant activation value is conveyed to the glomeruli during the time of odor presentation (Fig. 2, glomerular, OSN activity). Twenty simulated PNs integrate the glomerular computations; in the model, the oscillatory spiking activity of the PNs' responses to odorants (Fig. 2, PN activity, output), resulting from the integration of excitatory and inhibitory inputs, is transformed into an average output signal calculated as the average normalized spike rate of a given PN during the time of odor presentation. These activation values can be directly compared with the time-averaged activation values measured from PNs in calcium-imaging experiments (Sachse and Galizia 2002). In those experiments, a membrane-impermeable calcium-sensitive dye (fura-dextran) was locally injected into the PN tract leaving the AL. Therefore only the PNs were backfilled with dye and could thereafter be selectively visualized in the AL (relative activation values vary between 0 and 1 and are referred to as glomerular output pattern in this paper). Note that in the absence of glomerular computations, the PN activation pattern, after normalization, would be an exact image of the glomerular input pattern in the model. Model PN activation patterns in response to three odorants (1-hexanol, 1-octanol, 1-nonanol) were compared with those published for calciumimaging experiments (Sachse and Galizia 2002).

First, we created interglomerular projection patterns in which each heteroLN receiving excitatory input in a given glomerulus could invade all other glomeruli with equal probability (stochastic network; Figs. $1 C i$ and $3 A$, thus creating networks in which inhibition was uniformly distributed. In these simulations, we varied both the probability $P$ of an inhibitory connection between two glomeruli $(0.0<P<1.0$ with a step size of 0.25 ) as well as the synaptic strength $w$ of these connections $(0.0<w<1.0$ with a step size of 0.025$)$. Note that with $P=1$, this results in the special case of an amorphous network in which there is no spatial connectivity structure. We then compared the PN patterns in response to all three odorants to those obtained experimentally by correlating the average PN activation patterns to the glomerular output patterns obtained experimentally. The highest average correlation of $r=0.80$ (average $r$ 's ranged from 0.78 to 0.80 ) in response to all three stimulations was obtained at $P=0.5$ and $w=0.725$. Figure $3 A$ shows the comparison between simulated and experimentally measured glomerular output patterns in response to 1-hexanol.

We then created an interglomerular projection pattern in which simulated glomeruli received inhibitory inputs from morphologically adjacent glomeruli [using the numbering scheme of Galizia et al. (1999); morphological network, Figs. $1 C i i$ and $3 B]$. The synaptic weights $w$ of these connections were varied between $w=0.0$ (no interglomerular inhibition) and $w=1.0$ (maximal inhibition) with a step size of 0.025 ; the projection pattern was not varied. For each synaptic weight value, we created 50 networks that we stimulated with the glomerular input activity patterns corresponding to 1-hexanol, 1-octanol, and 1-nonanol. The average PN activation patterns evoked in response to each of these three glomerular input patterns (i.e., simulated odorants) were computed, normalized, and compared with experimentally obtained glomerular output activities by correlation. Thus for each synaptic weight value $w$, the normalized average $\mathrm{PN}$ activation patterns in response to all three inputs were correlated with the glomerular output patterns obtained experimentally and the "best" synaptic weight value was chosen to be that for which the overall strength of that correlation was highest. The best match with experimental data were obtained with weak interglomerular inhibition $(w=0.05)$; at this synaptic weight value, the average correlation between experimental and simulation data were $r=0.80$ (Pearson correlation coefficient). Average $r$ 's ranged from 0.22 to 0.80 over the range of synaptic weight values tested. Figure $3 B$ shows a comparison between experimental and simulated output activation patterns for 1-hexanol at the "best" synaptic weight value of 0.05 .

The best results were obtained when we created an interglomerular projection pattern based on functional correlations between glomerular input activity patterns to odorants (functional network, Figs. 1Ciii and 3C). Experimentally, glomerular input patterns were obtained in response to 60 odorants (Galizia et al. 1999; Sachse et al. 1999). We here considered the possibility that glomeruli would inhibit each other proportionally to the similarity of their olfactory response profiles, i.e., that a functional inhibitory network would exist. As a 
A stochastic network

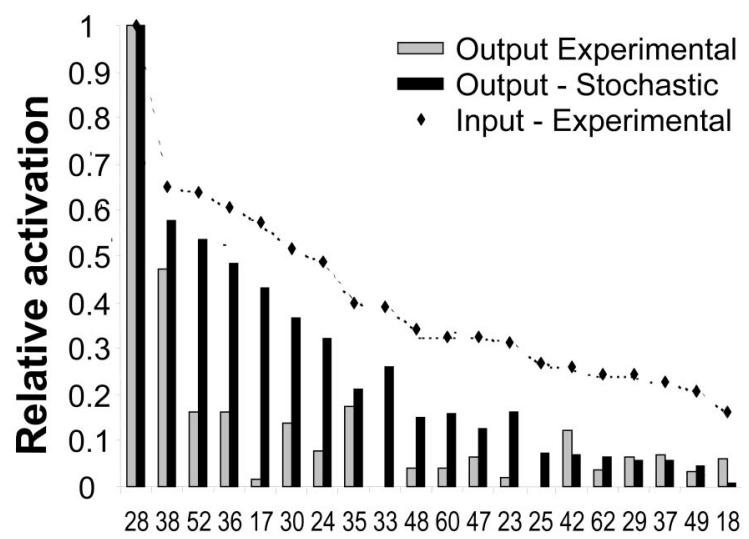

Glomerulus


Glomerulus

C functional network

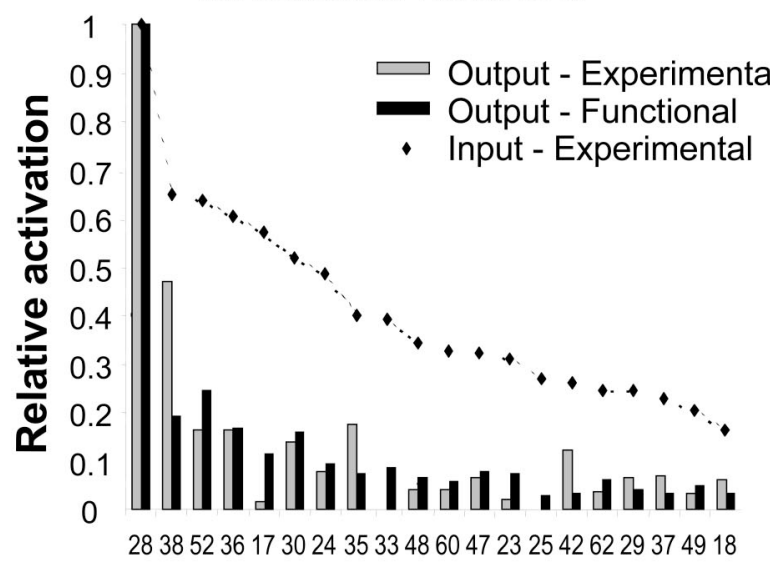

Glomerulus

FIG. 3. Simulated output activation patterns in response to stimulation of the model with 1-hexanol. The graphs compare the normalized glomerular output patterns measured experimentally (experimental-output) to those obtained in simulations using the stochastically (stochastic-output, $A$ ), morphologically (morphological-output, $B$ ) and functionally (functional-output, $C$ ) based interglomerular inhibition patterns. For comparison purposes, the normalized input activation patterns are also depicted (experimental-input). consequence, glomeruli activated by common groups of odorants would be more likely to inhibit each other than those activated by noncommon groups of odorants. To determine the possible inhibitory projection patterns, we used glomerular input activity data from the calcium imaging experiments in response to all 60 odors. The glomerular input patterns of the 20 glomeruli considered here were correlated pairwise, resulting in a single variable $c(-1<c<1)$ describing the olfactory response profile similarity between every pair of glomeruli. The positive values from this matrix were used directly to scale the inhibitory synaptic weights between pairs of glomeruli, whereas negative values were set to zero (Table 1); consequently, the inhibitory connections between any two glomeruli had synaptic strengths of $c^{*} w$ (with $c^{*} w \geq 0$ ). Using this established pattern of interglomerular inhibition, we varied $w$ between $w=0.0$ and $w=1.0$ with a stepsize of 0.025 and ran 50 simulations for each value of $w$. As described in the preceding text, the average resulting PN activation patterns were then correlated with the average glomerular output patterns from the experimental data. The maximal average correlation of $r=0.90$ between the simulated and experimentally measured PN responses to the three odor stimulations was obtained when the synaptic weight $w$ was set to $w=0.15$. The average values of $r$ obtained over the range of synaptic weight values chosen ranged from 0.75 to 0.90 . Figure $3 C$ shows the comparisons between the experimentally measured input and output patterns and the simulated PN activation patterns in response to 1-hexanol at this best value of $w$. In comparison (see Fig. 4A), the correlations between simulated and experimentally measured output patterns resulting from the functional inhibition network were significantly higher than those obtained with the morphological or stochastic inhibitory network [morphological: $F(1,4)=30.11, P<0.005$; stochastic: $F(1,4)=18.4, P<0.05$ ], whereas the latter two were not significantly different from each other $[F(1,4)=0.07$, $P>0.5]$.

As detailed in the preceding text, the degree of inhibition (strength of the synaptic weights) was varied systematically to determine the best fit with the data for each type of inhibitory network. As a consequence, the average activities across PNs varied from very low to very high depending on the strength of the synaptic weight for both the anatomically and functionally organized inhibitory networks. The SDs of the average PN activities across the range of synaptic weights used was 0.05 for both types of networks, strongly suggesting that the better "best fit" for the functionally organized network did not arise due to lower average PN activity only. In case of the stochastically constructed network (each LN can inhibit each glomerulus with equal probability), the inhibitory network tends to dampen the PN activities in a uniform manner. As a consequence, after normalization to the highest PN response, the SD of average PN activity across all synaptic weight amplitudes is very low (0.007). Fully connected networks, in which all glomeruli inhibited all others proportionally to their own activation strength (probability of connection equals 1, "amorphous" connections) did not replicate the experimentally measured PN activation patterns, suggesting the AL does not simply perform a suppression of the lesser activated glomeruli.

Comparisons of odor activity patterns at the glomerular input and output of the AL have suggested that the interglomerular network serves to decrease the correlations among 
A

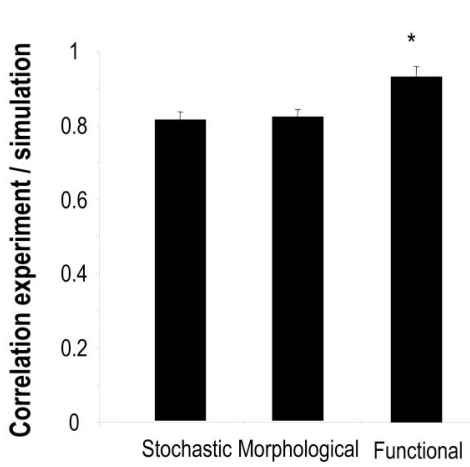

B



1

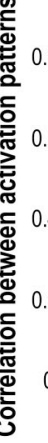

$-0.2$ $\square$ Experimental - Input

Stochastic Inhibition - Output

Morphological Inhibition - Output

Functional Inhibition Output

Experimental - Output$$
\text { (n) }
$$

(2)
tational models (morphological, stochastic, and functional). The correlations calculated for the functionally based interglomerular inhibition pattern are significantly ( $P<0.01$ by ANOVA) different from the others shown. $B$ : correlations between the activation patterns in response to pairs of odorants, experimental and simulations. The graph shows the correlations between time-averaged activation patterns in response to pairs of odorants: experimentalinput: correlation between time-averaged glomerular activation patterns measured by bath-application of calcium sensitive dyes (from Sachse et al. 1999); experimental-output: correlation between time-averaged $\mathrm{PN}$ activation patterns in response to 2 odorants measured by injection of calcium sensitive dyes in PNs (from Sachse and Galizia 2002); stochastic inhibition-output: correlation between time-averaged $\mathrm{PN}$-activation patterns in response to pairs of odorants resulting from simulations using interglomerular inhibitory projections chosen stochastically with a probability of 0.5 ; morphological inhibition-output: correlation between timeaveraged $\mathrm{PN}$-activation patterns in response to pairs of odorants resulting from simulations using interglomerular inhibitory projections based on morphological neighborhood relationships between glomeruli; functional inhibition-output: correlation between time-averaged PN-activation patterns in response to pairs of odorants resulting from simulations using interglomerular inhibitory projections based on similarity between glomerular odor response profiles. $C$ : simulation results for the 3 "best" networks when the glomerular input and output activations from a different experimental set including 3 odorants at multiple concentrations were compared. The graph shows the average correlations between simulated and experimentally measured $\mathrm{PN}$ activation patterns in responses to odorants at 6 different concentrations.

odor activation patterns (Sachse and Galizia 2002) (Fig. 4B, compare experimental input-output), presumably facilitating behavioral discrimination among odorants. Indeed, pairs of odorants for which PN responses were more strongly decorrelated (Sachse and Galizia 2002) were more readily discriminated by bees in a behavioral experiment than those for which PN responses were highly correlated (Laska et al. 1999). In our simulations, only the interglomerular inhibitory connections based on glomerular response profiles reduced the correlations between pairs of odorants at the glomerular output level with respect to their correlations at the glomerular input level (Fig. $4 B$, functional inhibition-output). Interestingly, AL networks with stochastic inhibitory connections reproduced the correlations between input activation patterns at the level of PN output (Fig. 4B, stochastic inhibition-input), whereas the network in which physically close glomeruli inhibited each other resulted in somewhat lower correlations between output activation patterns as compared with input activation patterns (Fig. 4B, morphological inhibition-output). These results indicate, as suggested previously (Linster and Cleland 2004), that to ensure contrast enhancement among odorants with similar and overlapping glomerular activation patterns, interglomerular inhibition should be constructed as a function of glomerular response profiles rather than on their physical proximity.

We then tested how our three "best" networks dealt with data from a different set of experiments, in which hexanol, octanol, and nonanol were each presented at six different concentrations (Sachse and Galizia 2003). These data were obtained under dissimilar conditions than those used in the previous simulations and are therefore ideally suited to test the generality of our results. To test how well the three "best" networks reproduced this new data set, we used the glomerular activation patterns in response to each of the 18 odorant stimulations ( 3 odorants at 6 different concentrations each) as input patterns to the AL network and compared the resulting PN activation patterns to those recorded experimentally. The network with functionally organized inhibition reproduced the experimentally measured input-output function significantly better than the two networks with stochastically and morphologically organized inhibition (functional compared with morphological $P<0.05$; functional compared with stochastic $P<$ 0.02; Fig. 4C). Interestingly, the average correlations with the experimentally recorded PN responses where best at intermediate concentrations $\left(10^{-1}\right.$ to $\left.10^{-3}\right)$, which corresponds to the working range of odor responses in PNs, i.e., to the steep part of their dose-response function. Correlations decreased considerably at very low concentrations $\left(10^{-5}\right)$, where odor-evoked patterns often consist of just a single activated glomerulus (Sachse and Galizia 2003). Whether the good performance of the morphologically designed network at these low concentrations is functionally relevant remains to be elucidated.

\section{I S C U S S I O N}

Our simulations show that the input-output function between sensory neuron and PN activation patterns provided by the 
honeybee AL network is best replicated when interglomerular inhibitory projections are organized along functional principles, that is, when interglomerular inhibition is proportional to the similarity in glomerular odor response profiles rather than dependent on physical distance.

The data that we used in the present study result from estimations of input activity derived from bath-applied dye (Sachse et al. 1999), whereas output activity estimates are measured as postsynaptic calcium increases (Sachse and Galizia 2003). Because the two techniques monitor different calcium responses, they cannot be compared directly. Rather, each response profile requires calibration across glomeruli as has been done here. We have avoided comparing input-output activity patterns directly; rather, we have determined the interglomerular network most likely to reproduce the experimentally observed transfer function by comparing simulated output with experimentally measured output.

We have previously argued that a positive functional correlation between inhibitory projection patterns and the underlying olfactory similarity space is necessary to render interglomerular inhibition an effective mechanism of contrast enhancement (Linster and Cleland 2004). This is in contrast to lateral inhibition in the retina, for example, in which neurons with similar (spatial) receptive fields are also physically adjacent, enabling effective contrast enhancement in these two physical dimensions. In the AL/OB network, odorants activate a wide, dispersed array of olfactory glomeruli (Galizia and Menzel 2001; Johnson et al. 1999), hence, lateral inhibition (in the classical sense in which neurons with similar response profiles are morphologically adjacent and inhibit one another) cannot exist; as a consequence, inhibition between glomeruli would necessarily be widely dispersed and be functionally rather than morphologically organized. This raises the question of whether efficient processing within the AL can depend on the spatial position of olfactory glomeruli. This view has been strongly advocated for the mammalian $\mathrm{OB}$, in which local interneurons may mediate a center-surround inhibitory network (Aungst et al. 2003).

In many brain areas, specific spatial arrangements optimize processing and/or total wiring length, a phenomenon best explored within the visual system. In this context, the organization of the inhibitory network correlates with response profiles of the neurons in that neurons responding to similar stimuli tend to inhibit each other more than those not responding to similar stimuli. It is the organization rather than the overall strength of the inhibitory connections that determines the functionality of the inhibitory network. In many systems, such as the visual system, response profiles can be predicted from physical location so that the organization of inhibitory interactions correlates with both physical proximity and response profiles ("lateral inhibition"). In a multidimensional olfactory world, however, two dimensions are not sufficient; hence, neighborhood relationships may be relevant for subgroups of glomeruli but not as a general rule. The simulation studies presented in this work strongly suggest that inhibitory connections within the AL are not spatially constrained and are not local, uniform, or stochastic but rather that inhibitory connections within the $\mathrm{AL}$ are dictated by the functional response properties of glomeruli. That is, with increasing overlap in their molecular response profiles, glomeruli increase the strength of inhibitory connections between them. Given the high-dimensional nature of olfactory representations, we speculate that similar principles will hold in the vertebrate OB. Additional mechanisms, some of which have been described in the OB, may also be involved in differentiating olfactory stimuli. For example, intraglomerular excitatory mechanisms (Schoppa and Westbrook 2002; Urban and Sakmann 2002) may contribute to enhancing the response of the most strongly activated glomeruli. To date, no excitatory synaptic interactions mediated by local interneurons have been described in the honeybee AL.

\section{A C K N O W LED G M EN T S}

The authors thank T. Cleland for comments on the manuscript.

\section{G R A N T S}

This work was supported by a grant from Human Frontier Science Program to C. G. Galizia and C. Linster.

\section{REFERENCES}

Aungst JL, Heyward PM, Puche AC, Karnup SV, Hayar A, Szabo G, and Shipley MT. Centre-surround inhibition among olfactory glomeruli. Nature 426: 623-629, 2003.

Christensen TA, Pawlowski VM, Lei H, and Hildebrand JG. Multi-unit recordings reveal context-dependent modulation of synchrony in odorspecific neural ensembles. Nat Neurosci 3: 927-931, 2000.

Cleland TA and Linster C. How synchronization properties among secondorder sensory neurons can mediate stimulus salience. Behav Neurosci 116: 212-221, 2002.

Duchamp-Viret P, and Duchamp A. GABAergic control of odour-induced activity in the frog olfactory bulb: possible GABAergic modulation of granule cell inhibitory action. Neuroscience 56: 905-914, 1993.

Faber T, Joerges J, and Menzel R. Associative learning modifies neural representations of odors in the insect brain. Nat Neurosci 2: 74-78, 1999.

Fonta C, Sun XJ, and Masson C. Morphology and spatial distribution of bee antennal lobe interneurons responsive to odours. Chem Senses 18: 101-119, 1993.

Galizia CG, McIlwrath SL, and Menzel R. A digital three-dimensional atlas of the honeybee antennal lobe based on optical sections acquired by confocal microscopy. Cell Tissue Res 295: 383-394, 1999.

Galizia CG and Menzel R. Odor perception in honeybees: coding information in glomerular patterns. Curr Opin Neurobiol 10: 504-510, 2000.

Galizia CG and Menzel R. The role of glomeruli in the neural representation of odors: results from optical recording studies. J Insect Physiol 47: 115-130, 2001.

Gascuel J and Masson C. A quantitative ultrastructural study of the honeybee antennal lobe. Tissue Cell 23: 341-355, 1991.

Hansson BS, Carlsson MA, and Kalinova B. Olfactory activation patterns in the antennal lobe of the sphinx moth, Manduca sexta. J Comp Physiol [A] 189: 301-308, 2003.

Joerges J, Kuettner A, Galizia G, and Menzel R. Representations of odors and odor mixtures visualized in the honeybee brain. Nature 387: 285-288, 1997.

Johnson BA, Woo CC, Hingco EE, Pham KL, and Leon M. Multidimensional chemotopic responses to n-aliphatic acid odorants in the rat olfactory bulb. J Comp Neurol 409: 529-548, 1999.

Laska M, Galizia CG, Giurfa M, and Menzel R. Olfactory discrimination ability and odor structure-activity relationships in honeybees. Chem Senses 24: 429-38, 1999 .

Linster $\mathbf{C}$ and Cleland TA. How spike synchronization among olfactory neurons can contribute to sensory discrimination. J Comput Neurosci 10 : 187-193, 2001.

Linster C and Cleland TA. Configurational and elemental odor mixture perception can arise from local inhibition. J Comput Neurosci 16: 39-47, 2004.

Linster $\mathbf{C}$ and Gervais R. Investigation of the role of interneurons and their modulation by centrifugal fibers in a neural model of the olfactory bulb. J Comput Neurosci 3: 225-246, 1996. 
Linster C and Hasselmo M. Modulation of inhibition in a model of olfactory bulb reduces overlap in the neural representation of olfactory stimuli. Behav Brain Res 84: 117-127, 1997.

Linster C, Johnson B, Yue E, Morse A, Xu Z, Hingco E, Choi Y, Choi M, Mehissa A, and Leon M. Perceptual correlates of neural representations evoked by odorant enantiomers. J Neurosci 21: 9837-9843, 2001.

Linster C and Masson C. A neural model of olfactory sensory memory in the honey bee's antennal lobe. Neural Comput 8: 94-114, 1996.

Linster C, Marsan D, Masson C, and Kerszberg M. Odor processing in the bee: a preliminary study of the role of central input to the antennal lobe. In Advances in Neural Information Processing Systems 6, edited by Cowan JD Tesauro G, and Alspector J. Morgan Kaufmann Publishers 1994, p. 527534.

Linster C and Smith BH. A computational model of the response of honey bee antennal lobe circuitry to odor mixtures: overshadowing, blocking and unblocking can arise from lateral inhibition. Behav Brain Res 87: 1-14, 1997.

MacLeod K and Laurent G. Distinct mechanisms for synchronization and tempora patterning of odor-encoding neural assemblies. Science 274: 976-979, 1996.

Malun D. Inventory and distribution of synapses of identified uniglomerular projection neurons in the antennal lobe of Periplaneta americana. J Comp Neurol 305: 348-360, 1991a.

Malun D. Synaptic relationships between GABA-immunoreactive neurons and an identified uniglomerular projection neuron in the antennal lobe of Periplaneta americana: a double-labeling electron microscopic study. Histochemistry 96: 197-207, 1991b.

Meister $\mathbf{M}$ and Bonhoeffer T. Tuning and topography in an odor map in the rat olfactory bulb. J Neurosci 15: 1351-1360, 2001.

Meredith M. Patterned response to odor in mammalian olfactory bulb: the influence of intensity. J Neurophysiol 56: 572-597, 1986

Ng M, Roorda RD, Lima SQ, Zemelman BV, Morcillo P, and Miesenbock G. Transmission of olfactory information between three populations of neurons in the antennal lobe of the fly. Neuron 36: 463-474, 2002.

Rubin BD and Katz LC. Optical imaging of odorant representations in the mammalian olfactory bulb. Neuron 23: 499-511, 1999
Sachse S and Galizia CG. Role of inhibition for temporal and spatial odor representation in olfactory output neurons: a calcium imaging study. $\mathrm{J} \mathrm{Neu}$ rophysiol 87: 1106-1117, 2002.

Sachse S and Galizia CG. The coding of odour-intensity in the honeybee antennal lobe: local computation optimizes odor representation. Eur J Neurosci 18: 2119-2132, 2003

Sachse S, Rappert A, and Galizia CG. The spatial representation of chemical structures in the antennal lobe of honeybees: steps towards the olfactory code. Eur J Neurosci 11: 3970-3982, 1999.

Schoppa NE and Westbrook GL. AMPA autoreceptors drive correlated spiking in olfactory bulb glomeruli. Nat Neurosci 5: 1194-1202, 2002.

Stopfer M, Bhagavan S, Smith BH, and Laurent G. Impaired odor discrimination on desynchronization of odour-encoding neural assemblies. Nature 390: 70-74, 1997.

Urban NN. Lateral inhibition in the olfactory bulb and in olfaction. Physiol Behav 77: 607-612, 2002.

Urban NN and Sakmann B. Reciprocal intraglomerular excitation and intraand interglomerular lateral inhibition between mouse olfactory bulb mitral cells. J Physiol 542: 355-367, 2002.

Wachowiak M, Cohen LB, and Zochowski MR. Distributed and concentration-invariant spatial representations of odorants by receptor neuron input to the turtle olfactory bulb. J Neurophysiol 87: 1035-1045, 2002.

Wachowiak M, Zochowski M, Cohen LB, and Falk CX. The spatial representation of odors by olfactory receptor neuron input to the olfactory bulb is concentration invariant. Biol Bull 199: 162-163, 2000.

Wang JW, Wong AM, Flores J, Vosshall LB, and Axel R. Two-photon calcium imaging reveals an odor-evoked map of activity in the fly brain. Cell 112: 271-282, 2003.

Wilson RI, Turner GC, and Laurent G. Transformation of olfactory representations in the Drosophila antennal lobe. Science 303: 366-370, 2004.

Xu F, Greer CA, and Shepherd GM. Odor maps in the olfactory bulb. J Comp Neurol 422: 489-495, 2000.

Yokoi M, Mori K, and Nakanishi S. Refinement of odor molecule tuning by dendrodendritic synaptic inhibition in the olfactory bulb. Proc Natl Acad Sci USA 92: 3371-3375, 1995 . 